Projets

de paysage

\section{Projets de paysage}

Revue scientifique sur la conception et l'aménagement de l'espace

9 | 2013

Le paysage a t-il imposé sa loi ?

\title{
La loi paysage a-t-elle eu un impact sur la planification territoriale?
}

L'exemple de l'évolution des projets de planification paysagère et des compétences au sein de la fonction publique

Impact of the Landscape Law on Territorial Planning - The Example of how Landscaping Planning Projects and Competencies are developing within the Public Services

\section{Didier Labat et Gaëlle Aggeri}

\section{OpenEdition}

\section{Journals}

\section{Édition électronique}

URL : http://journals.openedition.org/paysage/11953

DOI : 10.4000/paysage. 11953

ISSN : 1969-6124

\section{Éditeur :}

École nationale supérieure du paysage de Versailles-Marseille, Institut national des sciences appliquées Centre Val de Loire - École de la nature et du paysage, École nationale supérieure d'architecture et de paysage de Bordeaux, École nationale supérieure d'architecture et de paysage de Lille, Agrocampus Angers

\section{Référence électronique}

Didier Labat et Gaëlle Aggeri, « La loi paysage a-t-elle eu un impact sur la planification territoriale? », Projets de paysage [En ligne], 9 | 2013, mis en ligne le 20 décembre 2013, consulté le 10 décembre 2020. URL : http://journals.openedition.org/paysage/11953; DOI : https://doi.org/10.4000/paysage. 11953

Ce document a été généré automatiquement le 10 décembre 2020.

Projets de paysage 


\section{La loi paysage a-t-elle eu un impact sur la planification territoriale?}

L'exemple de l'évolution des projets de planification paysagère et des compétences au sein de la fonction publique

Impact of the Landscape Law on Territorial Planning - The Example of how Landscaping Planning Projects and Competencies are developing within the Public Services

Didier Labat et Gaëlle Aggeri

1 Nous proposons d'approfondir les retombées de la loi Paysage de $1993^{1}$ en abordant ses objectifs relatifs à la prise en compte du paysage dans les documents d'urbanisme et dans le pilotage des projets. Nos travaux s'appuient sur une analyse des politiques publiques, en particulier des instruments d'action publique (Lascoumes, 2004). Ils ont pour objectif d'étudier le lien entre la loi Paysage et la transformation des pratiques locales mais également l'évolution des compétences dans les administrations publiques, maîtres d'ouvrage de projets. Ainsi que le soulignent Jean-Pierre Le Bourhis et Pierre Lascoumes (2014), l'analyse de la résistance aux instruments permet de mieux comprendre les ressorts d'une politique publique et des agents chargés de la mise en œuvre des projets. L'objectif de planification par ou avec le paysage, issu de la loi Paysage, a logiquement conduit au développement des compétences et des métiers relatifs à ces expertises.

2 Mais cette transformation est-elle observable et peut-on la décrire ? Au-delà du politique, l'appropriation de l'objectif d'une planification par le paysage ne serait-elle pas favorisée par une structuration de ces compétences dans les organisations des instances publiques en charge des politiques paysagères et de planification? Et par là même, l'évolution de ces métiers n'est-elle pas un traceur des mutations des pratiques et des enjeux de l'action publique?

3 Nous traiterons de ces questions en proposant en premier lieu comme corpus d'étude une lecture synthétique des évolutions successives de la prise en compte du paysage dans les documents d'urbanisme sur le territoire girondin. Puis, nous proposons de 
brosser un portrait de la structuration des compétences dans les administrations publiques liées à cet exercice de pilotage du projet paysager. Nous fondons notre démonstration sur des études des évolutions des métiers d'État et des collectivités locales pour poser en perspective le devenir des métiers du paysage face à une action publique paysagère qui se diversifie et élargit ses horizons.

\section{Entre norme réglementaire et dynamique de projet, en quoi le paysage sert-il les territoires?}

4 Le concept de développement durable apparu peu avant la loi Paysage a favorisé l'ancrage de l'enjeu environnemental dans les nombreuses évolutions réglementaires de la planification territoriale. Mais face à l'urgence des enjeux de préservation des ressources naturelles, le paysage a-t-il trouvé sa place dans le projet de territoire ? Et si tel est le cas, de quelle façon s'est-il immiscé dans les pratiques de gestion et de planification des territoires? Nous témoignons ici du constat d'une mobilisation nouvelle des acteurs pour une interterritorialité au sein de laquelle le paysage semble prendre part comme socle fédérateur interdisciplinaire.

\section{De la loi de 1993 au Grenelle de l'environnement : un cadre normatif en constante mutation}

5 La loi Paysage apparaît dix ans après les lois de décentralisation des années 1982 et 1983. Elle est perçue par les acteurs de l'aménagement comme une loi d'urbanisme et d'aménagement et accompagne la montée en puissance du projet de planification territoriale que les différents types de collectivités apprennent à maitriser dans le cadre de leurs compétences respectives. Par son insertion dans les premiers articles du code de l'urbanisme (articles L. 110 et L. 121 et suivants en particulier), le paysage devient un objet d'intervention obligatoire dans les plans d'occupation des sols et les schémas directeurs. Le paysage est ainsi inséré dans des dispositifs de régulation des dynamiques spatiales sur l'ensemble du territoire. Mais au-delà d'une volonté vertueuse d'une planification par le ménagement, c'est toute une hiérarchie socioéconomique qui se joue par l'intermédiaire du zonage. Ces outils d'intervention spatiale ont été qualifiés "d'instrument neutralisé et détourné » par Jean-Pierre Le Bourhis et Pierre Lascoumes (2014) pour expliciter l'écart entre les objectifs du code de l'urbanisme et l'application de la norme sur le terrain.

6 Il faut attendre la loi Solidarité et Renouvellement urbain de 2000 (loi SRU) pour observer une multiplication de projets dans lesquels le paysage prend un nouvel essor que nous attribuons en particulier à trois phénomènes.

7 En premier lieu, dans les années 1990, le vaste chantier de rénovation des espaces publics, piloté par les collectivités (Donadieu, 2003), a participé d'une nouvelle vague culturelle des paysages urbains. Répondant à la demande sociale croissante et à une volonté politique de mise en scène de l'espace partagé, l'intervention paysagère s'est imposée sans obligation réglementaire en matière de qualité des paysages des villes. Selon des démarches de marketing territorial urbain, la valorisation des espaces publics devient une politique de différenciation des villes et de reconstruction d'une centralité fonctionnelle et attractive. Par exemple, depuis le réaménagement des quais en rive 
gauche par le paysagiste Michel Corajoud, la ville de Bordeaux accueille dans ces lieux les fêtes du fleuve et du vin (Tapie et al., 2009).

Ensuite, la loi SRU impulse une nouvelle dynamique territoriale liée à la transformation des outils de la planification. Les POS et schémas directeurs sont transformés en plans locaux d'urbanisme (PLU) et en schémas de cohérence territoriale (Scot). Les règles de non-constructibilité par défaut d'outil de planification entrânent nombre de territoires à s'engager dans une démarche de développement urbain dans un contexte de métropolisation et d'étalement des pôles secondaires. Les périphéries s'organisent et l'éclosion des Scot suit le développement de l'intercommunalité, elle-même promue par la loi Chevènement ${ }^{2}$. Le document d'urbanisme devient ainsi, au-delà du zonage, un outil de marketing et de mise en concurrence des territoires (Pinson, 2004). Une sorte de translation scalaire se produit entre les stratégies urbaines locales et la pensée du développement des grands territoires (communes et intercommunalités). Dans ce mouvement, le paysage devient à son tour une ressource à investir pour individualiser les territoires et se distinguer (Pecqueur, 1998, Larrue et al., 2013). Là encore, cette évolution répond à une demande sociale croissante pour un cadre de vie renouvelé dans les cœurs urbains mais aussi au-delà des villes centres dans les périphéries qui sont à leur tour concernées par la planification intercommunale.

Enfin, les outils de planification ne s'élaborent plus uniquement à partir de la somme des projets locaux pour répondre aux exigences des pétitionnaires. Les procédures d'élaboration se complexifient et sont investies d'évaluations environnementales issues de l'application de la directive européenne sur l'évaluation environnementale des plans et programmes ${ }^{3}$. Il en ressort de nouvelles normes d'élaboration du projet de territoire : l'environnement est moins une contrainte d'aménagement et davantage un enjeu réglementaire à respecter pour limiter tout contentieux administratif et retard dans la mise en œuvre des règles de constructibilité. Cependant, par manque de définition juridique, le paysage reste encore un objet d'intervention plutôt mobilisé par la rhétorique planificatrice (et politique) que par une réelle intervention matérielle dans la planification (Monédiaire, 2003). Entre protection et préservation, le paysage émerge mais peine à être affirmé. C'est le thème de la biodiversité qui, à partir de 2007 dans le cadre du Grenelle de l'environnement, se voit porteur des enjeux liés aux paysages dans la traduction des trames vertes et bleues. D'autres politiques sectorielles mobilisent également le paysage, que ce soit par l'intermédiaire des énergies renouvelables, des déplacements ou de l'agriculture par exemple. Ainsi, le paysage semble devenir peu à peu un outil de médiation sociale entre acteurs et usagers mais aussi un outil fédérateur de l'action publique avec une capacité de mise en cohérence des politiques sectorielles. C'est d'ailleurs un des fondements de la Convention européenne du paysage (CEP) ${ }^{4}$ qui, moins de dix ans après la loi Paysage de 1993, a insufflé un nouvel ordre dans la qualification du terme de "paysage ». Ouverte sur les perceptions des habitants, la définition donnée au paysage s'est résolument tournée vers une approche culturaliste et holistique, contrairement aux approches naturalistes de l'écologie du paysage.

10 Cette lecture des évolutions réglementaires trouve écho à l'observation des dynamiques d'acteurs. Nous constatons un retour à des pratiques d'intégration des approches environnementales sectorielles pour penser le territoire dans sa globalité et sa complexité. Le paysage constitue alors un des maillons opérationnels ou le socle de ces pratiques. Le territoire girondin en est un exemple singulier que nous avons investi à 
partir d'une démarche exploratoire d'évaluation des politiques paysagères dans les documents d'urbanisme.

\section{Le paysage mobilisé comme ciment du projet de territoire : l'exemple de la commande publique dans la métropole bordelaise}

11 Pour argumenter l'hypothèse d'une mutation de l'action paysagère, nous nous appuyons sur une recherche portant sur l'évaluation a posteriori de la politique paysagère du schéma directeur (SD 2001) de l'aire métropolitaine de Bordeaux (Labat et Donadieu, 2013) $)^{5}$. L'analyse historique de la planification sur ce territoire met en relief plusieurs dynamiques territoriales permettant d'esquisser des conséquences de la loi Paysage de 1993. Les atlas des paysages qu'elle a impulsés ont garanti des modalités de projets fédératrices de valeurs collectives ${ }^{6}$. En reconnaissant leurs paysages, les acteurs $\mathrm{du}$ territoire ont construit les outils d'une dialectique de l'espace géographique mais aussi social. Dans le cas bordelais, ce fut la conception de l'atlas des paysages de la Gironde en 1997 qui a eu un impact important sur la planification stratégique métropolitaine (Folléa et Gautier, 1997). L'équipe de paysagistes et d'urbanistes Follea et Gautier a conçu dans ce contexte de décentralisation le premier référentiel sur les grands paysages piloté par l'État. Cet atlas a conduit à un changement méthodologique de construction du projet territorial du Sysdau ${ }^{7}$, structure intercommunale en charge du futur schéma directeur, devenu $\operatorname{Scot}^{8}$ depuis la loi SRU. Sur les bases de l'atlas, un schéma directeur pour les paysages métropolitains a été produit par la même équipe en 1999 et a permis de fonder la « charpente paysagère » du SD 2001. Cet outil est devenu, pour le Sysdau, un outil pour gérer des conflits internes liés aux tensions intercommunales entre le centre et la périphérie, et pour raisonner d'une manière plus collective et projective les droits au développement.

Cette nouvelle dialectique du projet de territoire par l'intervention paysagère a participé à la construction d'une identité du Sysdau et à ses fondations politiques. Les élus se sont reconnus dans un discours qui renvoie à leur quotidien ainsi qu'à celui des habitants, au-delà d'une vision " technocratique » des documents de planification. Dans ce cas précis, l'impact important de la loi Paysage est lié aux outils de connaissance mais aussi d'appartenance spatiale selon des mécanismes cognitifs. Un effet levier indéniable peut lui être imputé dans notre cas d'étude et il se poursuit par un effet domino au sens de l'action publique locale.

Peu après, la communauté urbaine de Bordeaux (CUB) élabore son PLU et lance à son tour une réflexion sur les paysages qui se construit d'abord sur une définition de la nature en ville, anticipant ainsi les lois Grenelle. Cette construction d'une culture paysagère communautaire gagne les territoires et s'inscrit dans un cadre multiscalaire de la planification en résonance avec les politiques urbaines.

Cependant, l'évaluation de la politique paysagère du SD 2001, que nous avons menée, témoigne des difficultés encore inhérentes à la mise en œuvre d'orientations paysagères. Malgré un engagement innovant et séduisant, elles n'ont pas été toutes suivies pour des raisons politiques et techniques. La transformation du SD 2001 en Scot constitue une nouvelle étape traduisant les effets de la loi SRU. Les objectifs d'une planification pilotée par projet conduisent le Sysdau à s'interroger sur l'application du Scot. Une nouvelle étape est franchie pendant les travaux du syndicat sur les trames vertes et bleues. Lors de leur définition, les logiques scientifiques et techniques d'une 
matérialisation spatiale de la biodiversité métropolitaine ont été refusées par les élus. Rigidité des inventaires scientifiques et pression d'étude non homogène sur l'ensemble du territoire ont été considérées comme inacceptables par les membres du Sysdau. Ils y ont vu un recul de leur part décisionnelle et ont préféré une proposition argumentée selon les logiques paysagères déjà engagées en 2001 qui leur ont permis de reprendre pied dans la construction des choix politiques. Dans cette situation, le paysage s'impose comme un outil de médiation entre les représentations identitaires de l'espace par les acteurs locaux et celles plus scientifiques de la biodiversité dans les trames vertes et bleues par l'État. En ce sens et de manière plus générale, le paysage apparait être l'expression spatialisée et incarnée d'une projection politique des acteurs décisionnaires (Bédard, 2009). Mais cette nouvelle génération de Scot est articulée selon une approche plus complète, mais aussi sectorielle (place de l'agriculture, trames vertes et bleues, économie forestière, etc.). Le paysage est ici mobilisé comme un métaoutil dans une dimension holistique régulatrice des tensions territoriales. C'est également ce qui est observable à l'échelle intercommunale dans le cadre de la charte paysagère (2004) de la communauté de commune de Montesquieu'. Cette intercommunalité fait partie du Sysdau et du Scot. Suite au SD 2001, elle a conçu sa propre charte destinée en particulier à la territorialisation de la charpente paysagère métropolitaine selon un ensemble de prescriptions plus opérationnelles. De manière itérative, elle est mobilisée dans le cadre de la révision du $\operatorname{Scot}^{10}$ en 2011, comme un outil des revendications en matière d'aménagement sous forme ascendante des attentes locales.

15 Enfin, la révision de l'atlas des paysages en 2011 constitue un nouvel exemple de l'impact de la loi Paysage de 1993, à corréler avec l'apport de la CEP. Le conseil général de la Gironde a repris le pilotage de cet atlas en poursuivant des démarches innovantes : révision de l'atlas des paysages, évaluation de sa mise en œuvre, politique paysagère assise sur la $\mathrm{CEP}$, rationalisation des subventions via les objectifs de l'atlas, etc. Cette fois-ci, l'atlas des paysages s'inscrit au cœur d'une stratégie d'acteurs. $\mathrm{Ne}$ disposant pas de compétence propre en matière de planification, le conseil général mobilise l'atlas comme référentiel pour argumenter et légitimer son intervention. De manière indirecte, cet atlas vient à nouveau nourrir la révision du Scot métropolitain de Bordeaux et est mis à disposition des autres structures de Scot dans le département ou par l'intermédiaire de la démarche InterScot. Le conseil régional aquitain vient à son tour reconnaître la place des paysages dans les enjeux qu'il porte à l'occasion des premières rencontres régionales sur l'InterSCOT qu'il a lui même initiées en janvier $2013^{11}$. Le processus de percolation des préoccupations sur les paysages suit son cours.

16 Ces travaux sur la planification inspirent de manière incrémentale les politiques plus locales. La mise en œuvre opératoire des intentions paysagères sur les grands territoires est au cœur des préoccupations ascendantes des gouvernements urbains. Par exemple, la démarche des « 55000 hectares de nature », initiée par la CUB ${ }^{12}$, vise la valorisation de terres non bâties pour préserver un capital de "nature", multifonctionnel et au service des habitants de la métropole. Pour répondre à un enjeu de transformation des pratiques, les élus ont initié un dialogue compétitif en faisant intervenir cinq équipes pour l'aide à la définition de stratégies d'intervention (gouvernance, économie locale, biodiversité, usages, etc.). Ici, les paysagistes sont mobilisés pour concevoir des politiques paysagères qui tendent à intégrer une approche systémique. Elle se traduit de manière verticale par la cohérence entre les outils d'intervention (du Scot à la parcelle), et horizontale en considérant les réponses 
possibles issues d'approches pluridisciplinaires (hydraulique, agriculture, économie territoriale, sociologie, politique, etc.).

Ces exemples Girondins montrent les avancées favorisées par la loi Paysage, notamment par les outils de connaissance des paysages. De manière intermittente, sporadique et indirecte, les outils élaborés à l'occasion de cette politique s'immiscent peu à peu comme référentiels et catalyseurs d'une vision partagée des enjeux des territoires. Bien entendu, la loi Paysage n'est pas seule responsable de cette évolution. Mais de nouvelles formes de politiques paysagères s'élaborent, agissant de manière incrémentale et cognitive sur la façon de discuter et d'élaborer les projets de territoires. Ce développement des politiques paysagères locales a-t-il une répercussion significative sur les porteurs de projets? Autrement dit, le paysage émerge-t-il parce qu'il s'impose par la norme et la loi ou bien parce que des hommes de l'art insufflent un changement?

\section{L'évolution des métiers et les perspectives pour les porteurs de projets publics de paysage}

L'analyse des compétences au sein des structures publiques est rarement mise en avant contrairement à celle de l'ingénierie privée mobilisée par des fédérations ou syndicats professionnels. Pourtant, le développement de ces compétences témoigne de l'adaptation de l'action publique aux politiques publiques sur les paysages.

19 Notre étude s'appuie sur la mise en perspective des répertoires des métiers de l'État et des collectivités locales et des données concernant le recrutement, les formations des maitres d'œuvre et d'ouvrage publics en paysage.

\section{L'adaptation des métiers du paysage dans les structures de décision et de commande publique : donnée objective ou flou généralisé ?}

De façon générale, peu de chercheurs se sont intéressés à la question de l'adéquation entre les attentes sociétales aujourd'hui mieux connues et l'adaptation des compétences et des métiers du paysage dans les services territoriaux et de l'État qui participent de la commande publique. Pierre Donadieu, en particulier, a dressé un portrait de ces compétences au sein de la société de manière générale (Donadieu, 2002, 2009).

21 Cependant, étant insuffisamment défini, le périmètre des interventions sur les paysages reste encore flou et n'aide pas à l'inventaire des métiers et des effectifs dans la fonction publique. Dans le secteur privé, les compétences des paysagistes et leur utilité dans l'exercice de production de la ville sont reconnues (Masboungi, 2002, 2011). Mais qu'en est-il dans le secteur public? Quels sont les profils et trajectoires des porteurs de projets de paysage?

\section{L'évolution des métiers du paysage et des recrutements dans les ministères comme traceur des attentes de l'État}

Les répertoires nationaux des métiers Rime, Rome, $\mathrm{Apec}^{13}$ ont bien pris en compte et formalisé le métier de concepteur-paysagiste (niveau de qualification Bac +5) pour des employeurs variés, privés mais aussi publics et parapublics : collectivités territoriales, 
conseils d'architecture, d'urbanisme et de l'environnement (CAUE), État en région, gestionnaires d'espaces naturels protégés. En interne, le répertoire des emplois types du ministère du Développement durable et de l'Énergie (MEDDE) et du ministère de l'Égalité des territoires (MET) fait apparaître les compétences en paysage dans six familles de métier, soit l'équivalent de dix emplois types, depuis l'inspecteur des sites, jusqu'au référent routier ou l'expert en biodiversité. Ce constat témoigne d'une diffusion de ces compétences dans plusieurs maîtrises d'ouvrage spécialisées. Mais plus largement au sein de l'État, l'inventaire reste complexe; d'une part, parce que les fonctions du paysagiste ne sont pas directement orientées vers la conception paysagère mais davantage vers la conduite de l'action publique; d'autre part, parce que le paysage est un sujet d'intervention dans nombre de ministères et services de l'État: établissements d'enseignement supérieur, universités, centres de recherche, services déconcentrés et centraux des différents ministères, etc.

Depuis quelques années, les ministères de l'Agriculture et de l'Écologie recrutent des paysagistes dans la filière technique des ingénieurs de travaux ${ }^{14}$. Ces paysagistes présents dans ces ministères officient essentiellement comme inspecteurs des sites dans les Direction régionale de l'environnement, de l'aménagement et du logement (Dreal), chargés de la planification territoriale en Direction Départementale des territoires (DDT), paysagistes dans le réseau scientifique et technique ou comme chargés de mission dans les services centraux. Pour autant, ils pourront occuper des postes de chargés d'études ou de mission plus transversaux concernant par exemple l'urbanisme ou l'environnement, mais rarement dans des postes clés de la décision publique. Faute d'analyse, leur nombre reste méconnu mais on peut l'estimer à une cinquantaine d'agents si l'on s'en tient aux paysagistes sortis d'écoles spécialisées. Pourtant, de nombreux autres professionnels du paysage exercent dans ces services, issus de formations universitaires par exemple. Il faut bien entendu, comptabiliser les paysagistes conseils de l'État (122 en activité en 2011), professionnels privés, qui œuvrent pour des missions publiques dans chaque département et région de France. Leur organisation associative ${ }^{15}$ leur permet d'être fédérés et de bénéficier d'une meilleure visibilité.

De façon générale, depuis les lois du Grenelle et leurs corollaires de plan «nature en ville» et de trame verte et bleue, on peut observer en interne parmi les chargés de mission et d'études en paysage d'État, un glissement perceptible des missions orientées vers l'écologie du paysage et les continuités vertes. Cette remarque fait écho à la représentation générale de ces métiers que le MEDDE a diffusée en 2012 à travers sa commande du répertoire des "métiers de la biodiversité » appliqué à tous les secteurs économiques (réalisation Atelier technique des espaces naturels, Association nationale pour la formation professionnelle des adultes) qui ne mettra en exergue que deux seuls métiers du paysage (concepteur-paysagiste et chargé de mission paysage) parmi les 39 métiers contribuant à la préservation de la biodiversité, réduisant le paysage à sa dimension écologique en laissant pour compte son apport patrimonial, social et culturel.

\section{Une évolution des métiers territoriaux du paysage au plus près des initiatives politiques locales}

Parallèlement, le répertoire des métiers de la fonction publique territoriale est porté et réactualisé par le Centre national de la fonction publique territoriale (CNFPT) tous les 
trois ans, sur la base d'une consultation d'un comité de pilotage de représentants de collectivités d'échelles variées. Il est soumis à l'avis du Conseil national d'orientation du CNFPT composé de représentants d'élus et de syndicats territoriaux qui apportent une légitimité politique et représentative du personnel. Ce répertoire revêt une dimension stratégique car il s'est imposé comme la référence des directions des ressources humaines qui s'en inspirent pour bâtir les fiches de postes de recrutement en fonction des politiques locales conjoncturelles. Certains métiers émergent, disparaissent ou évoluent dans leurs compétences chaque année.

La fiche de concepteur-paysagiste a été créée en 2000 dans la famille " espaces verts » (85 000 agents). Il est souvent paysagiste de formation et opère essentiellement dans les bureaux d'études de métropoles, notamment dans les services espaces verts de villes, espaces publics des agglomérations, routes des conseils généraux. Les dernières statistiques de l'Observatoire du CNFPT de 2005 donnent approximativement 200 concepteurs-paysagistes, de statut technicien (niveau Bac +2 ou 3) ou ingénieur territorial (niveau master scientifique ou ingénieur), recrutés majoritairement dans les années 1990-2000. Dans un contexte actuel majoritaire d'externalisation des études d'espaces publics à des bureaux d'études privés, la suppression de ce métier ou son intégration comme spécialisation d'autres métiers plus représentatifs ont été débattues dans la sphère des décideurs et du groupe de pilotage lors des révisions du répertoire CNFPT en 2009 et 2013 (Aggeri, 2009 et 2014). Serait-il alors une spécialisation de chef de projet aménagement urbain, chef de projet paysage, chef de projet développement local ? Malgré ces débats entre élus et experts, ce métier, très faiblement représenté, est cependant encore conservé en raison de son aspect stratégique pour l'image des villes et des argumentaires de poids des paysagistes mobilisés dans le comité de pilotage : cœur de métier spécifique, niche d'acteurs stratégiques, spécificité du dessin. Les compétences dans la fiche métier « concepteur-paysagiste » de la version 2009 ont évolué en 2012 pour prendre en compte les savoir-faire d'écologie urbaine, de concertation et de coproduction avec les habitants. Si les écoles supérieures du paysage mettent en France toujours l'accent sur les formations de landscape architect et designer, les analyses précédentes de l'État comme des collectivités convergent pour montrer que les besoins de concepteurs-paysagistes, en capacité de proposer un projet de paysage aux décideurs, sont rares en 2013 dans la fonction publique. Seuls quelques professionnels experts sont réunis dans des collectivités à culture paysagère forte, notamment dans les services espaces verts et publics des grandes villes (Montpellier, Lyon) ou d'intercommunalités (Nice métropole), pour porter des projets de qualification des espaces publics en complément des concours de paysage destinés à des bureaux d'études privés.

Le métier "chef de projet de paysage", quant à lui, relève de la famille de l'environnement (14560 agents en 2011) au même titre que les métiers transverses de l'éducation au développement durable et des études environnement. Il est intégré au répertoire CNFPT en 2005 suite à une enquête qualitative au sein de ses employeurs potentiels qui révèle en 2006, environ 100 cadres A et B spécialisés dans le pilotage d'études, le conseil et la mise en cohérence des politiques paysagères locales dans les documents d'urbanisme (Stocker, 2008). Il exerce essentiellement dans les intercommunalités de petite et grande taille pour la maitrise d'ouvrage des projets d'infrastructures vertes (trames vertes et bleues, lignes de tramways et circuits doux, entrées de ville, agriculture urbaine...), la commande et la coordination de schémas 
directeurs de paysage pour des écoquartiers, quartiers ANRU, ZAC, et la mise en cohérence des diagnostics paysagers avec les documents d'urbanisme du Scot, PLU, plans climat énergie territoriaux (PCET), Agendas 21.Il peut aussi travailler dans des grands territoires au sein d'un parc naturel régional pour le conseil des collectivités rurales lors de la mise en œuvre des plans et chartes du paysage ou au sein des départements et régions pour coordonner un atlas du paysage, un schéma directeur " éolien et paysage " par exemple. Ces dernières années, ces métiers sont souvent devenus hybrides avec la fusion de postes qui adoptent de nouveaux intitulés : « chef de projet urbanisme et paysage » ou " chef de projet trame verte et bleue et paysage ». On peut relever une émergence de ce métier du point de vue des effectifs et des recrutements en hausse mais aussi de celui de leurs missions stratégiques qui apportent une plus-value au territoire sur le plan du tourisme, de l'identité territoriale et de la qualité de vie.

Trois postures différentes des chefs de projet paysage sont mises en avant selon les commandes et ambitions politiques des territoires : celle du maitre d'ouvrage chef de projet transversal (aménagement des espaces publics, développement local, aménagement du territoire) qui utilise le matériau du paysage comme socle fédérateur de politiques sectorielles plus larges; celle du chef de projet en ingénierie écologique travaillant sur l'écologie du paysage qui se rapproche de la version État «métiers de la biodiversité » portée par le MEDDE ; celle de l'ingénierie sociale œuvrant à mobiliser les habitants par des outils de représentation du paysage (observatoires photographiques, expositions, enquêtes...), à coproduire des projets de paysage avec les habitants, voire à fédérer des initiatives citoyennes autour de projets environnementaux (agriculture urbaine, villes zéro pesticide, jardins partagés). Ainsi en 2012, la fiche de ce métier a été réactualisée en valorisant les compétences liées aux continuités écosystémiques, aux sciences sociales et à l'agriculture urbaine. Ces conclusions sur l'évolution des missions des chefs de projet en paysage dans les structures d'État et territoriales sont en adéquation avec l'évolution des politiques publiques citées plus haut vers une "grenellisation" d'une part, et vers un développement d'une maitrise d'ouvrage transversale des projets à la double échelle des villes et des grands territoires ruraux et naturels, d'autre part.

\section{Mais quels sont les profils et compétences initiales et professionnelles de ces chefs de projet spécialisés en paysage dans les collectivités ?}

Les entretiens exploratoires du CNFPT confortés par une étude des profils des candidats aux concours territoriaux montrent que certains d'entre eux sont des diplômés ingénieurs du paysage des écoles d'Angers, de Blois ou de l'école d'architecte de Bordeaux, seuls admis à passer le concours d'ingénieur territorial (niveau master scientifique et technique).

Le seul itinéraire de formation professionnelle de paysage, commun à la fonction publique d'État et territoriale, organisé par l'ATEN/CNFPT depuis 2008, constitue un traceur des trajectoires des hommes porteurs de ces politiques publiques. Dans le module fondamental de "Lecture de paysage, outils de diagnostics, représentations, concertation " et le module "Nature en ville : maîtrise d'ouvrage ", les participants, nombreux, relèvent de parcours et de responsabilités variés. Et ils mobilisent périphériquement les notions de paysage comme outils de médiation. On peut compter parmi eux des acteurs territoriaux comme des directeurs de services espaces verts, 
fonciers, urbanisme, systèmes d'information géographiques (SIG), environnement, développement local, tourisme, transport, énergie dans des territoires d'échelles variées (villes, départements, régions, PNR). On note en parallèle des métiers d'État chargés de mission par exemple en Parc national, Conservatoire du littoral, Voie navigable de France, Réseau ferré de France. Le module de formation experte «Politique publique en paysage : charte et plan paysage » n'est mis en œuvre que rarement faute d'inscrits (CNFPT, 2003 à 2013).

Paradoxalement, si le domaine du paysage reste une référence partagée par les acteurs $\mathrm{du}$ projet public, les concepteurs-paysagistes de la fonction publique sont moins mobilisés que les bureaux d'études privés sinon dans des projets à forte imprégnation en écologie urbaine. Des maitres d'ouvrage généralistes de l'environnement, du développement durable, de l'aménagement urbain et rural sont privilégiés pour mobiliser de préférence des savoirs de paysage comme socles assembleurs de disciplines sectorielles et d'outils de concertation et de médiation sociale.

\section{Quelles perspectives pour les porteurs de projets dans la fonction publique?}

Nous faisons le constat que finalement le paysage est utilisé par les acteurs pour ce qu'il suscite dans l'inconscient collectif. Le cas bordelais permet de souligner qu'il sert également à compenser les blocages inhérents aux instruments d'action publique. Au nom du paysage, le politique se réapproprie son rôle d'arbitre devant les multiples enjeux parfois contradictoires que la planification renferme, et les techniciens l'utilisent pour englober des sujets conflictuels ou techniquement aporiques. La loi ne suffit pas pour engager une modification massive des pratiques des acteurs sur le paysage. C'est davantage le développement toujours croissant des politiques sectorielles qui nécessite un outil syncrétique, besoin auquel le paysage semble au moins en partie répondre.

Pour autant, cet exemple de politiques paysagères multiscalaires sur la métropole bordelaise est-il isolé ou étendu sur d'autres territoires? Sans enquête appropriée, il est pour le moment difficile d'y répondre. Un inventaire serait utile vu le contexte mobilisateur. Les projets de lois 2013 sur la " réforme des collectivités territoriales » portant sur la reconfiguration des compétences selon les typologies de collectivités (intercommunalités, communes, Établissent public territorial de bassin [EPTB] ...) sont l'occasion d'interroger la cohérence des multiples politiques publiques qui ont un impact direct, indirect, ou cumulé sur les paysages. La planification territoriale des Scot ou des PLU intercommunaux paraît une échelle pivot favorable à l'intégration des politiques sectorielles dans un même espace de réflexion et d'action. La prise en compte du paysage dans ces outils constitue un enjeu méthodologique certain pour la gouvernance des paysages. Car c'est bien la question de la coordination des différentes politiques de l'État et des collectivités territoriales qui est posée. L'actualité le démontre.

L'État s'investit actuellement dans la rédaction d'un projet de loi-cadre sur la biodiversité, laissant une place secondaire au paysage mais renouvelant une partie des objectifs de la loi Paysage. Peut-on en conclure que la loi Paysage n'aurait pas atteint ses objectifs ? Il faut davantage intégrer cette volonté dans un exercice de déploiement 
des objectifs de la CEP, en grande partie fondés par des experts ayant collaboré à la loi Paysage (Luginbühl, 2012).

Par ailleurs, des réseaux d'acteurs régionaux, souvent coanimés par les Dreal et les organismes techniques et scientifiques spécialisés (CAUE, écoles du paysage, universités), se développent et témoignent d'initiatives locales collectives de l'action publique. Par exemple, la Dreal Midi-Pyrénées, conjointement avec les partenaires locaux ${ }^{16}$, anime un réseau "Paysage» (créé dans les années 2000), rassemblant quelques 250 personnes de multiples horizons, notamment des chercheurs, des aménageurs et décideurs des collectivités et de l'État. Des actions de sensibilisation, de formation opérationnelle ou d'information sont régulièrement organisées pour fédérer le réseau.

Cette "mise en tension » des acteurs nous parait contribuer à un inventaire de la loi Paysage et constitue une orientation certaine pour répondre aux enjeux actuels et à venir. En effet, les incertitudes économiques, l'évolution des modes de vie et des recompositions sociodémographiques modifient les territoires. Ne serait-il pas nécessaire d'anticiper ces évolutions et d'inscrire le paysage comme sujet prospectif ? Dans ce cadre, il nous semble important de réinterroger l'action publique dans cet objectif d'anticipation et de réflexivité nécessaire pour réagir, et préparer l'avenir. Les 40 années passées d'étalement urbain ont marqué les esprits et montré tout l'enjeu d'une préservation des paysages, capital et ressource économique majeurs pour la France. Les exercices de prospective de la Datar (Datar, 2011), du MEDDE, de l'Ademe (scénarios énergétiques 2030 et 2050), ceux menés en région par les collectivités ou l'État ne devraient-ils pas investir les paysages de demain comme cela est demandé par les habitants du monde rural et urbain, ou comme le projettent les experts de la transition écologique dans une société postcarbone à faible empreinte écologique? Des travaux tels que « Territoires 2040 » de la Datar l'intègrent au même titre que les scénarios de métropole-nature à biodiversité positive du MEDDE (étude Biopolis, Commissariat général au développement durable, 2012) mais de façon encore sporadique et peu communicante. Or, la couverture nationale du territoire par des Scot d'ici $2017^{17}$ constitue une opportunité parmi d'autres, pour traduire ces réflexions prospectives sur les territoires, dans un rapport au quotidien entre le global et le local.

\section{Conclusion}

La loi Paysage a fortement participé à l'essor du paysage dans les instruments d'action publique en France. Son impact n'est pas nécessairement issu de son poids réglementaire, mais plutôt des outils de connaissance qui ont permis une première appropriation de nos paysages. Les lois qui suivirent dans les années 2000 ont davantage conduit les acteurs à mobiliser le paysage comme outil intégrateur d'une somme de préoccupations sectorielles. Enfin, la CEP a défini le paysage et propose une structuration de l'action publique paysagère favorable à une gouvernance des paysages. La dimension sensible, présente dans la définition et non réglementaire, constitue une perspective performative et cognitive qui mérite d'être analysée. Dans le contexte de remise en question du millefeuille administratif, nous vivons peut-être une période transitoire. Après de fortes pressions en faveur de la biodiversité, vient le temps d'une gestion plus concertée et complémentaire entre les représentations des populations en faveur du cadre de vie et de la valorisation des paysages et l'enjeu prioritaire national 
de préservation de la biodiversité. L'État et les collectivités ne gagneraient-ils pas à interroger leurs actions selon un modèle cumulatif pour en discerner l'efficacité et les impacts?

Les métiers du paysage peuvent contribuer à cet exercice de gouvernance dans leurs évolutions de compétences en faveur des sciences sociales. Il n'y a peut-être plus intérêt à savoir qui fait quoi ou à cantonner les paysagistes dans des missions strictes de projets paysagers, mais à comprendre comment les compétences du porteur de projets de paysage se diffusent et répondent aux attentes sociétales et politiques. Un des apports de la loi Paysage est d'avoir justement participé à cette évolution en replaçant les démarches participatives de manière indirecte dans le creux de l'action publique. En développant les étapes de la connaissance des paysages et en élaborant des outils d'action ou de projet, la loi Paysage a conduit en anticipation de la CEP à reconfigurer l'action paysagiste. Sans statut professionnel précis et sans reconnaissance institutionnelle dans les administrations publiques, les nouvelles compétences acquises par les pilotes de démarches paysagères ont tout de même participé au renouvellement d'une ingénierie de projet sur les territoires, depuis l'analyse des grands espaces jusqu'à l'espace public et la médiation de proximité. Mais, sans évaluation formelle de cette politique, il est difficile de quantifier et de qualifier précisément son impact sur le développement des métiers du paysage.

\section{BIBLIOGRAPHIE}

Aggeri, G., « Études sur l'évolution des métiers territoriaux, famille espaces verts et environnement », CNFPT, 2013, 2009, 2005, 15 p.

Barthon, C., Garat, I., Gravari-Barbas, M. et Veschambre, V., «L'inscription territoriale et le jeu des acteurs dans les événements culturels et festifs : des villes, des festivals, des pouvoirs ", Géocarrefour, vol. 82/3, 2007, p. 111-121.

Bédard, M., Le Paysage, un projet politique, Québec, Presses de l'université du Québec, 2009, 330 p.

CNFPT, « Répertoire des métiers territoriaux, Fiches métiers », 2005 et 2009, 300 p.

Datar, «Territoires 2040. Des systèmes spatiaux en perspective », Revue d'études et de prospective, $\mathrm{n}^{\circ} 3,2011,175 \mathrm{p}$.

Donadieu, P., La société paysagiste, Arles/Versailles, Actes Sud/ENSP, 2002, 149 p.

Donadieu, P. et Dumont-Fillon, N. « Les processus de mise en œuvre des politiques publiques de paysage en milieu urbain : intérêts, limites et perspectives de développement des actions conçues avec le concours des paysagistes », rapport final - Programme de recherche « Politiques publiques et paysages : analyse, évaluation, comparaison », MEDD, 2003, 124 p.

Donadieu, P., Les Paysagistes ou les Métamorphoses du jardinier, Arles/Versailles, Actes Sud/ENSP, $2009,169 \mathrm{p}$. 
Folléa, B. et Gautier, C. «Connaissance et valorisation des paysages de la Gironde. (Étude préalable à la définition d'une politique du paysage) », DDE de la Gironde, Diren Aquitaine, 1997, $270 \mathrm{p}$.

Kociemba, V., Roy, C. et Velasco-Graciet, H., « La vigne, le vin et la ville. Expériences bordelaises d'une trilogie vécue ", Sud-Ouest européen, vol. 22, 2006, p. 13-25.

Labat, D. et Donadieu, P., « Le paysage, levier d'action dans la planification territoriale », L'Espace géographique, vol. 1, t. 42, 2013, p. 44-60.

Larrue, C., Bonnefond, M., Gerber, J.-D. et Knoepfel, P., « Ressource paysagère et territoire : une nécessaire régulation?", dans Luginbühl, Y, Terrasson, D. (dir.), Paysage et Développement durable, Versailles, Éditions Quae, 2013, p. 129-142.

Lascoumes, P. et Le Galès, P., Gouverner par les instruments, Paris, Les Presses SciencesPo., coll. «SciencePo Gouvernances », 2004, 370 p.

Le Bourhis, J.-P. et Lascoumes, P., « Les résistances aux instruments de gouvernement. Essai d'inventaire et de typologie des pratiques ", dans Halpern, C., Lascoumes, P., et Le Galès, P. (dir.), L'Instrumentation et ses effets, Les Presses ScienesPo., Paris, 2014.

Luginbühl, Y., La Mise en scène du monde. Construction du paysage européen, Paris, CNRS, 2012, 432 p.

Masboungi, A. (dir.), Le Paysage en préalable. Michel Desvigne. Grand prix de l'urbanisme 2011. Joan Busquets, Prix spécial, Marseille, Éditions Parenthèses et DGALN, coll. « Grand Prix de l'urbanisme/ MEDDTL », 2011, 125 p.

Masboungi, A., Penser la ville par le paysage, Paris, Éditions de La Villette, coll. « Projet urbain », $2002,96 \mathrm{p}$.

Monédiaire, G., « La prise en compte du paysage dans les instruments de planification en droit français ", Revue juridique de l'environnement, vol. 3, 2003, p. 278-300.

Pecqueur, B., « Le projet de développement local : stratégie des firmes et stratégie des territoires ", dans Cuillier, F. (dir.), Les Débats sur la ville, Bordeaux, Éditions Confluences/a'urba., 1998, p. 103-112.

Pinson, G., « Le projet urbain comme instrument d'action publique », dans Lascoumes, P. et Le Galès, P. (dir.), Gouverner par les instruments, Les Presses ScienesPo., Paris, 2004, p. 199-233.

Pinson, G., « Projets de ville et gouvernance urbaine. Pluralisation des espaces politiques et recomposition d'une capacité d'action collective dans les villes européennes ", Revue française de science politique, vol. 56, 2006, p. 619-651.

Stocker C., « Enquête sur la représentativité des paysagistes dans les collectivités locales », rapport de fin d'année commandé par le CNFPT Montpellier, École nationale supérieure de la nature et du paysage de Blois, 2008, $35 \mathrm{p}$.

Tapie, G., Masson, C. et Mauvoisin, M., « Fabriquer la ville. 1995-2008 : espaces publics bordelais », dans Godier P., Sorbets C. et Tapie G. (dir.), Bordeaux Métropole. Un futur sans rupture, Marseille, Éditions Parenthèses, coll. « La ville en train de se faire », 2009, p. 150-173.

Verdier, J.-J., « La démarche paysagère territoriale et les “Grenelles” de l'Environnement », dans https://topia.fr/, septembre 2009. 


\section{NOTES}

1. Loi Paysage $n^{\circ}$ 93-24 du 8 janvier 1993 sur la protection et la mise en valeur des paysages et modifiant certaines dispositions législatives en matière d'enquêtes publiques. La loi instaure en particulier dans la planification des objectifs de préservation de la qualité des paysages dans le POS et de protection d'éléments de paysage à l'article L. 123-1-7 du code de l'urbanisme. À une échelle plus large, la politique nationale d'atlas des paysages est développée pour améliorer la connaissance des paysages dès 1994 .

2. Loi du 12 juillet 1999 relative au renforcement et à la simplification de la coopération intercommunale.

3. Circulaire d'application de la directive en France : circulaire $n^{\circ} 2006-16$ UHC/PA2 du 6 mars 2006 relative à l'évaluation des incidences de certains documents d'urbanisme sur l'environnement, MTETM, DGUHC, SAU.

4. Cf. le site suivant du Conseil de l'Europe : http://conventions.coe.int/Treaty/FR/treaties/html/ 176.htm

5. La démarche d'évaluation a été menée dans le cadre d'une thèse et selon 4 axes d'analyse : une analyse historique et documentaire des processus de planification depuis les années 1950, une interprétation sémiologique et structurelle du schéma directeur de l'aire métropolitaine de Bordeaux complétée par des entretiens d'acteurs, deux enquêtes à l'échelle métropolitaine et communale auprès d'habitants pour mieux comprendre les effets des politiques planificatrices sur les représentations paysagères.

6. Le poids de l'atlas des paysages a été révélé dans le cadre de la trentaine d'entretiens individuels menés dans la recherche et par l'analyse historique et de contenu des multiples études engagées par les acteurs.

7. Le Sysdau, syndicat du schéma directeur d'aménagement et d'urbanisme, est créé en 1996. Il répond à la nouvelle organisation des compétences des collectivités issue des lois de décentralisation. La révision du SDAU de 1980 constitue alors la première expérience du Sysdau sans l'action directe de l'État en matière de planification stratégique.

8. Le SD 2001 a été mis en révision en 2007 pour être transformé en Scot, dans les objectifs du Grenelle de l'environnement. Faisant parti des premiers « Scot Grenelle », il a été mobilisé dans le cadre de la démarche nationale Scot Grenelle pilotée par le MEDDE et de nombreux partenaires.

9. Dans le cadre d'une analyse des cohérences externes de la politique paysagère du SD 2001, les chartes et plans de paysage disponibles sur le territoire d'étude ont été analysés pour identifier les liens explicites ou implicites avec le SD 2001. Il s'agissait de vérifier l'efficacité des prescriptions du SD 2001, par conformité, compatibilité ou simple résonance cognitive des stratégies identifiées.

10. Dans le cadre des avis des personnes publiques associées, la communauté de communes de Montesquieu a produit un avis entièrement construit à partir de la charte paysagère pour revendiquer une modulation des objectifs du Scot.

11. La démarche InterScot initiée par le conseil général de la Gironde sert de levier au conseil régional d'Aquitaine pour revendiquer sa participation aux décisions d'aménagement, en l'absence d'une planification régionale. Le paysage ne dispose pas d'une entrée aussi fédérative à l'échelle régionale. Il sert cependant à revendiquer un droit à la cohérence des politiques locales avec les grands programmes du conseil régional, que ce soit par le biais de sa politique touristiques, culturelle ou économique. Cet engagement de la Région s'appuie sur l'association Pays et Quartiers d'Aquitaine qu'elle finance.

12. La CUB regroupe 28 communes et quelques 720000 habitants. Dans le cadre des projets métropolitains, la CUB a initié plusieurs démarches participatives à différents stades : réflexion prospective, choix de projets, débats publics, etc., dont celui des « 55000 hectares de nature ». 
13. Rime : répertoire interministériel des métiers de l'État (édition 2010); Rome : répertoire des métiers des pôles emploi ; Apec : Répertoire des métiers des cadres.

14. Plusieurs concours de recrutement sur titre ont été organisés afin d'intégrer des paysagistes dans les services de l'État sous le statut d'ingénieur des travaux publics de l'État (MEDDE) et, depuis cette année, sous le statut d'ingénieurs de l'agriculture et de l'environnement (MAP). Ce concours est ouvert à tous les paysagistes justifiant d'un diplôme de niveau master.

15. Cf. le site dédié : http://www.paysagistes-conseils.org/

16. Il s'agit de l'Union régionale des CAUE, des laboratoires Géode (Toulouse le Mirail) et Cepage (École nationale supérieure d'architecture et de paysage de Bordeaux), de l'École nationale de formation agronomique de Toulouse.

17. La loi Grenelle II impose une couverture nationale par des Scot à compter du 1er janvier 2017. Cet engagement est amorcé par la dynamique des Scot ruraux ayant fait l'objet d'un appel à projet lancé par le MEDDEe en 2011.

\section{RÉSUMÉS}

L'article questionne l'impact de la loi Paysage sur les démarches de planification spatiale et sur l'évolution des compétences au sein de la maîtrise d'ouvrage publique. Les exemples développés illustrent en effet les modalités d'émergence d'une nouvelle planification paysagère entreprise à l'initiative des collectivités, au plus près de la demande et de la participation citoyenne, et la mobilisation nouvelle dans le cadre des évolutions des compétences d'acteurs diversifiés du paysage, experts ou initiés. L'analyse montre qu'au-delà d'une meilleure intégration du paysage dans les outils de planification, l'évolution des métiers des porteurs de projets témoigne davantage d'un conflit de représentations entre les impératifs issus du Grenelle de l'environnement et le renforcement des compétences transverses de la maîtrise d'ouvrage publique. Au final, la prise en compte des paysages ne dépend pas seulement de mesures législatives et le changement de l'action publique concerne avant tout l'adaptation continue des acteurs aux attentes des sociétés quant à leur cadre de vie et leurs usages.

We question the impact of the Landscape Law, concerning procedures of spatial planning and the evolution of competencies among public project owners. The examples developed indeed illustrate how a new landscape planning is emerging at the initiative of collectivities, while conforming as closely as possible to citizen requests and participation and relying upon a new mobilization within the framework of the changing competencies of divers landscape actors, experts and people in the know. Our analysis shows that in parallel with better use of the landscape in planning tools there is also growing evidence of a conflict of representation between the requirements rooted in the Grenelle of the environment and the reinforcement of cross-cutting competencies in the field of project management among the trades involving project promoters. In the end, inclusion of the landscape is not simply a matter of legislative measures. Ultimately changes in public action imply above all that the professionals involved adapt themselves to societal expectations concerning the living environment and habits. 
INDEX

Mots-clés : paysage, planification, métiers, compétences, maîtrise d'ouvrage publique

Keywords : landscape, urban planning, profession, competence, public project owner

\section{AUTEURS}

\section{DIDIER LABAT}

Didier Labat est paysagiste DPLG de l'ENSP, urbaniste OPQU et docteur de l'AgroParisTech/ENSP Versailles. Il exerce au sein du Centre d'études techniques de l'Équipement du Sud-Ouest comme chargé de mission «Évaluation et prospective ». Il est chercheur associé au Laboratoire du paysage (Larep). Ses préoccupations de recherche portent sur l'évaluation des politiques publiques, l'action publique sur les paysages et la planification territoriale.

Didier.Labat[at]developpement-durable.gouv[dot]fr

\section{GAËLLE AGGERI}

Gaëlle Aggeri est ingénieur paysagiste de l'INH et docteur de l'AgroParisTech/ENSP Versailles. Ingénieur en chef territorial, elle a exercé 12 ans comme concepteur-paysagiste en collectivité locale puis 10 ans comme responsable du pôle de compétence national ingénierie écologique du CNFPT/INSET Montpellier. Elle est chercheur associé au Laboratoire du paysage (Larep). Ses sujets de recherche portent sur l'analyse des politiques publiques de paysage et de nature et des représentations sociales des décideurs et des usagers.

Gaelle.AGGERI[at]cnfpt[dot]fr 